Check for updates

Cite this: Mater. Adv., 2020, 1,77

Received 13th February 2020 Accepted 30th March 2020

DOI: 10.1039/d0ma00026d

rsc.li/materials-advances

\title{
High doping efficiency in p-type Al-rich AlGaN by modifying the $\mathrm{Mg}$ doping planes
}

\author{
Xinjia Qiu, (D) Yingda Chen, Enze Han, Zesheng Lv, Zhiyuan Song and Hao Jiang*
}

\begin{abstract}
High doping efficiency of the $\mathrm{Mg}$ dopant in Al-rich AlGaN is highly desired for AlGaN based deep ultraviolet optoelectronics. In this work, the Mg doping planes were modified by a pulsed group-III source, according to first-principles calculation results on $\mathrm{Mg}$ substituting $\mathrm{Al}$ or $\mathrm{Ga}$ on the surface and in the bulk of Ai-rich AlGaN. The Mg-delta-doped $\mathrm{Al}_{x} \mathrm{Ga}_{1-x} \mathrm{~N}(x \sim 0.42)$ layer, using a pulsed Ga source to modify the doping planes, exhibits a very high hole concentration of $8.3 \times 10^{18} \mathrm{~cm}^{-3}$, which is $67 \%$ higher than that of the reference one. A record doping efficiency of $51.9 \%$ was achieved and the consequent resistivity is as low as $0.51 \Omega \mathrm{cm}$. It was found that the $\mathrm{Mg}$ incorporation was significantly enhanced and the acceptor activation energy $\left(E_{A}\right)$ was substantially reduced, contributing to the high doping efficiency. The improved incorporation is attributed to the enhanced $\mathrm{Mg}$ substitution for $\mathrm{Al}$ at the doping planes and the increased substitution for $G a$ in the bulk by the modification, whereas the decreased $E_{\mathrm{A}}$ is ascribed to the enlarged valence-band bending. This method provides a simple and effective way to realize efficient p-type doping of Al-rich AlGaN.
\end{abstract}

\section{Introduction}

Ternary AlGaN alloys have been recognized as promising materials for realizing deep ultraviolet (DUV) optoelectronic devices with operating wavelengths down to $200 \mathrm{~nm} .{ }^{1-3}$ For the development of high performance AlGaN-based DUV devices, high-conductivity p-type Al-rich $\mathrm{Al}_{x} \mathrm{Ga}_{1-x} \mathrm{~N}(x \geq 0.4)$ is essential. Many studies have shown that enhancing the p-type conductivity has a significant effect on the improvement of both the electrical and optical properties of AlGaN DUV optoelectronics. ${ }^{4-8}$ However, achieving efficient p-type doping in Al-rich AlGaN is still a great challenge for the following reasons: (i) the limited solubility of magnesium (Mg), which is caused by the high formation enthalpies of $\mathrm{Mg}$ substitution for $\mathrm{Ga}$ or $\mathrm{Al}$; (ii) the high activation energy for Mg-doped $\mathrm{Al}_{x} \mathrm{Ga}_{1-x} \mathrm{~N}$ increasing from $\sim 170$ to $630 \mathrm{meV}$ for $x=0$ to $x=1$, due to the low valence band maximum, the deep impurity levels, and the heavy effective mass of the holes; and (iii) the strong self-compensation arising from the presence of donor-like native defects and/or complexes. ${ }^{9}$ These factors have led to the fact that the doping efficiency of the p-type Al-rich AlGaN reported in most literature is usually less than or around $1 \% .{ }^{5,10,11}$

So far, a number of p-type doping approaches, including superlattice (SL) doping, ${ }^{12} \mathrm{Mg}$ delta $(\delta)$ doping, ${ }^{13}$ polarizationinduced hole doping, ${ }^{7}$ and $\mathrm{Mg}$-Si alternative co-doping, ${ }^{14}$ have

State Key Laboratory of Optoelectronic Materials and Technologies, School of Electronics and Information Technology, Sun yat-sen University, Guangzhou 510275, People's Republic of China. E-mail: stsjiang@mail.sysu.edu.cn been developed to improve the p-type doping efficiency of Al-rich AlGaN. From the perspective of the mechanism, these techniques were mainly focused on reducing the acceptor ionization energy by energy-band modulation. There are few reports, however, on the efficient incorporation of the $\mathrm{Mg}$ dopant. It is known that the solubility of impurities can be enhanced near a semiconductor surface such as $\mathrm{Si}(001)$, because the energy of impurities is reduced due to the inherent stress relief near the surface. ${ }^{15}$ Such surface effects are therefore expected to be utilized to improve the incorporation efficiency of the Mg dopant in Al-rich AlGaN.

Delta doping is a long established approach to spatially confine dopants by interrupting the growth of the matrix material to make the dopants deposit on the exposed surface during epitaxial growth. In this method, the doping layer is divided into multiple periodic bulk layers and temporary surfaces, thus providing conditions for making full use of the surface and bulk effects of dopant impurity incorporation. In this work, first-principles calculations were utilized to analyze the behaviors of substituting $\mathrm{Mg}$ for $\mathrm{Ga}$ and $\mathrm{Al}$ on the surface and in the bulk of AlGaN. On the basis of the calculation results, it was proposed to improve the incorporation efficiency of the $\mathrm{Mg}$ dopant in Al-rich AlGaN by modifying the $\mathrm{Mg}-\delta-$ doping planes (i.e., doping surface) with a pulsed group-III source. The p-type doping efficiency was significantly improved in the AlGaN epilayer by modifying the $\delta$-doping planes with a pulsed Ga source. The effects of pulsed group-III sources on the $\mathrm{Mg}$ incorporation on the doping plane and in the bulk were investigated in detail. The mechanisms for the efficiency 
improvement were interpreted mainly in terms of dopant incorporation and activation energy. Furthermore, the applicability of this doping strategy was verified by the fabrication of all Al-rich AlGaN pn junctions.

\section{Experimental section}

\section{Computational method}

The first-principles calculations of total energies were performed within the framework of density functional theory (DFT), implemented in the Vienna ab initio simulation package (VASP). ${ }^{16,17}$ The interactions between valence electrons and ion cores were treated by Blöchl's all-electron-like projector augmented wave (PAW) method. ${ }^{18}$ The electronic exchange and correlation were described in the generalized gradient approximation (GGA), using the Perdew-Burke-Ernzerhof (PBE) functional. ${ }^{19}$ A cutoff energy of $425 \mathrm{eV}$ was used for the plane-wave basis set, while the $\mathrm{Ga}\left(3 \mathrm{~d}^{10} 4 \mathrm{~s}^{2} 4 \mathrm{p}^{1}\right), \mathrm{Mg}\left(3 \mathrm{~s}^{2}\right), \mathrm{Al}$ $\left(3 \mathrm{~s}^{2} 3 \mathrm{p}^{1}\right)$, and $\mathrm{N}\left(2 \mathrm{~s}^{2} 2 \mathrm{p}^{3}\right)$ states are treated as valence electrons. Bulk calculations were conducted using a $3 \times 3 \times 3$ supercell with 108 atoms and the Brillouin zone was sampled on a $3 \times 3 \times 3 k$-point Monkhorst-Pack mesh. ${ }^{20}$ In the case of the surface, a $(3 \times 3)$ supercell consisting of a six-bilayer thick slab along with a vacuum layer of $\sim 12 \AA$ was used to describe the AlGaN(0001) surface with a $3 \times 3 \times 1 k$-point mesh. The three bottom $\mathrm{Al}_{x} \mathrm{Ga}_{1-x} \mathrm{~N}$ bilayers were fixed in the appropriate bulkoptimized configuration to simulate the surface growth, while all the atoms were fully relaxed in the bulk calculations. Convergence with respect to the plane-wave cutoff energy and $k$-point sampling has been carefully checked. The convergence criterion for the forces of structure relaxations is $0.01 \mathrm{eV} \AA^{-1}$.

In the calculations, the formation energy $\left(E^{f}\right)$ of $\mathrm{Mg}$ was the main object of our research as $E^{\mathrm{f}}$ is a key factor for the $\mathrm{Mg}$ incorporation efficiency. Generally, $E^{\mathrm{f}}$ of neutral $\mathrm{Mg}$ impurities can be calculated by ${ }^{21}$

$$
E^{\mathrm{f}}[\mathrm{Mg}]=E_{\mathrm{tot}}[\mathrm{Mg}]-E_{\mathrm{host}}-\sum_{i} n_{i} \mu_{i},
$$

where $E_{\text {tot }}[\mathrm{Mg}]$ and $E_{\text {host }}$ are the total energies of the AlGaN supercell with and without $\mathrm{Mg}$ substitution, $n_{i}$ indicates the number of atoms of type $i$ (host or impurity atoms) that have been added to $\left(n_{i}>0\right)$ or removed from $\left(n_{i}<0\right)$ the supercell when the impurity is created, and $\mu_{i}$ is the corresponding chemical potentials of these species. The chemical potentials vary depending upon the chemical environment and can be given as

$$
\begin{gathered}
\mu_{\mathrm{Al} / \mathrm{Ga}}=\mu_{\mathrm{AlN} / \mathrm{GaN}}-\mu_{\mathrm{N}}, \\
3 \mu_{\mathrm{Mg}}+2 \mu_{\mathrm{N}}=\mu_{\mathrm{Mg}_{3} \mathrm{~N}_{2}} .
\end{gathered}
$$

Eqn (2) and (3) allow us to relate $\mu_{\mathrm{Mg}}$ and $\mu_{\mathrm{Al} / \mathrm{Ga}}$ to $\mu_{\mathrm{N}}$. Combining this information with eqn (1), we obtain the relationship between $E^{\mathrm{f}}[\mathrm{Mg}]$ and the concerned parameters, which is

$$
E^{\mathrm{f}}[\mathrm{Mg}]=E_{\mathrm{tot}}[\mathrm{Mg}]-E_{\mathrm{host}}+\mu_{\mathrm{AlN} / \mathrm{GaN}}-\frac{1}{3} \mu_{\mathrm{N}}-\frac{1}{3} \mu_{\mathrm{Mg}_{3} \mathrm{~N}_{2}},
$$

where $\mu_{\mathrm{AlN} / \mathrm{GaN}}$ and $\mu_{\mathrm{Mg}_{3} \mathrm{~N}_{2}}$ are the enthalpy of formation of AlN (GaN) and $\mathrm{Mg}_{3} \mathrm{~N}_{2}$, respectively, and are constants. ${ }^{22,23}$

\section{Growth and characterization of p-AlGaN epitaxial films}

The AlGaN epilayers were grown on 2 inch $c$-plane sapphire substrates by the low-pressure metalorganic chemical vapor deposition (MOCVD) method. Trimethyl-gallium (TMGa), trimethyl-aluminum (TMAl), trimethyl-indium (TMIn), and ammonia $\left(\mathrm{NH}_{3}\right)$ were used as precursors for $\mathrm{Ga}, \mathrm{Al}, \mathrm{In}$, and $\mathrm{N}$, respectively, while bis-cyclopentadienyl magnesium $\left(\mathrm{Cp}_{2} \mathrm{Mg}\right)$ was employed for p-type doping. Hydrogen was used as a carrier gas through the whole growth run. The basic epi-structure consists of a $400 \mathrm{~nm}$-thick AlN layer, an about $350 \mathrm{~nm}$-thick $\mathrm{Mg}$ - $\delta$-doped $\mathrm{Al}_{x} \mathrm{Ga}_{1-x} \mathrm{~N}(x \sim 0.4)$ layer grown at $1080{ }^{\circ} \mathrm{C}$, and an $8 \mathrm{~nm}$-thick Mg-doped GaN cap layer with a doping level of $\sim 7 \times 10^{19} \mathrm{~cm}^{-3}$ for an ohmic contact. A control sample was at first grown by the $\mathrm{Mg}$ - $\delta$-doping method with In as a surfactant, in which the group-III sources and $\mathrm{Cp}_{2} \mathrm{Mg}$ were alternately opened and closed, while $\mathrm{NH}_{3}$ was open all the time. ${ }^{24}$ As a surfactant, the flow rate of TMIn was kept at the optimized value of $140 \mathrm{sccm}$ during the growth period of $20 \mathrm{~nm} \mathrm{Al}_{x} \mathrm{Ga}_{1-x} \mathrm{~N}$ $(x \sim 0.4)$. An optimized V/III ratio of 3140 was used for depositing AlGaN. In the process of $\delta$ doping, the $\mathrm{NH}_{3}$ flow was held constant, which is equivalent to an infinite V/III ratio condition (i.e., an extremely $\mathrm{N}$-rich condition). The second sample was obtained by introducing a pulsed TMGa flow (PTGF) into the doping process, right after the $48 \mathrm{~s} \mathrm{Mg- \delta}$-doping step in each doping period. The third sample was grown with the same recipe, except that the PTGF was replaced by a pulsed TMAl flow (PTAF). The duration time of the PTGF or PTAF was set at an optimized value of $4 \mathrm{~s}$. Fig. 1 outlines the source flow sequence during one period of the modified $\delta$-doping process and the schematic epitaxial structure of the $\mathrm{Mg}$ - $\delta$-doped AlGaN sample grown with the PTGF.

After the growth, all the three samples were thermally annealed at $730{ }^{\circ} \mathrm{C}$ for $25 \mathrm{~min}$ in an $\mathrm{N}_{2}$ atmosphere to dissociate the $\mathrm{Mg}-\mathrm{H}$ complex. Temperature-dependent Hall-effect measurements were carried out to characterize the electrical properties of the p-type samples using the standard Van der Pauw geometry. Circular ohmic contacts with a diameter of $0.5 \mathrm{~mm}$ were formed by depositing a Ni/Au metal stack followed by annealing at $550{ }^{\circ} \mathrm{C}$ in an oxygen atmosphere for $2 \mathrm{~min}$. During the measurements, the part of the p-GaN cap layer outside the ohmic contacts was removed to eliminate the effect of parallel conduction. Secondary ion mass spectroscopy (SIMS) measurements were performed to investigate the depth profiles of the $\mathrm{Al}$ and $\mathrm{Ga}$ secondary ionic intensity and $\mathrm{Mg}$ atomic concentration in the three samples. Transmittance spectra of the deposited p-AlGaN layers grown on double-side polished (0001) sapphire substrates were recorded using a Shimadzu UV2550 ultravioletvisible spectrophotometer. The $\mathrm{Al}$ composition and structural crystalline quality were determined by high-resolution X-ray diffraction (HRXRD) measurements using a Bruker D8 Discover instrument.

\section{Fabrication and characterization of the AlGaN PN diode}

Full Al-rich AlGaN pn-junctions were fabricated and characterized to verify the feasibility of the p-type Al-rich AlGaN layer 


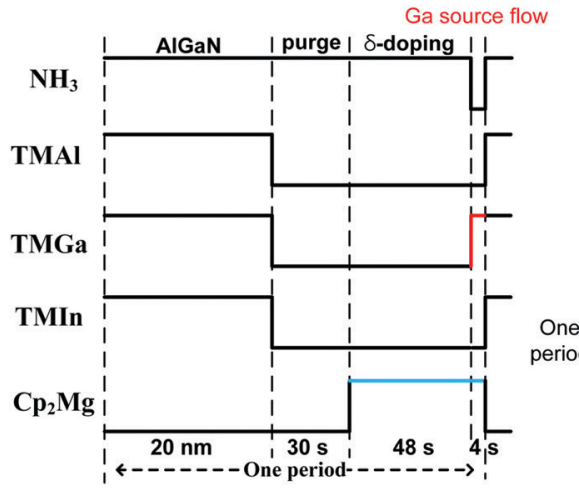

(a)



(b)

Fig. 1 Flow sequence of the modified $M g-\delta$ doping process with In as a surfactant (a) and the schematic side view of the epitaxial structure of the p-AlGaN grown with the PTGF (b).

grown with the modified $\mathrm{Mg}$ - $\delta$-doping plane. The epitaxial structure comprises a $500 \mathrm{~nm}$-thick optimized high-temperature AlN buffer layer, a $360 \mathrm{~nm}$-thick Si-doped $\mathrm{Al}_{0.4} \mathrm{Ga}_{0.6} \mathrm{~N}$ layer $\left(n \sim 2.7 \times 10^{18} \mathrm{~cm}^{-3}\right.$ ), and a $170 \mathrm{~nm}$-thick p-type $\mathrm{Al}_{x} \mathrm{Ga}_{1-x} \mathrm{~N}$ $(x \sim 0.4)$ layer deposited by the PTGF method. Device processing was started with circular mesa formation using conventional photolithography and dry-etching down to the $\mathrm{n}-\mathrm{Al}_{0.4} \mathrm{Ga}_{0.6} \mathrm{~N}$ layer. After the surface treatment, n-type ohmic contacts made of a $\mathrm{Ti} / \mathrm{Al} / \mathrm{Ni} / \mathrm{Au}$ metal stack were deposited by electron-beam evaporation and alloyed by a rapid thermal annealing process at $830{ }^{\circ} \mathrm{C}$ in an $\mathrm{N}_{2}$ atmosphere for $30 \mathrm{~s}$. The p-type ohmic contacts were made by depositing Ni/Au and annealed at $550{ }^{\circ} \mathrm{C}$ in an ambient atmosphere. Currentvoltage $(I-V)$ characteristics of the fabricated devices were measured using a Keithley 4200-SCS semiconductor characterization system under dark and UV-illuminated conditions. The illumination was supplied by a UV light emitting diode with a peak wavelength of $266 \mathrm{~nm}$.

\section{Results and discussion}

The incorporation behavior of the $\mathrm{Mg}$ dopant in the $\mathrm{Mg}-\delta$ doped $\mathrm{Al}_{x} \mathrm{Ga}_{1-x} \mathrm{~N}(x \sim 0.4)$ epitaxial layer, in which the $\mathrm{Al}$ composition is typically $\sim 40 \%$ in the bulk and $\sim 80 \%$ at the $\delta$-doping plane, ${ }^{24}$ was at first investigated using first-principles calculations. In the calculations, the value of the $\mathrm{N}$ chemical potential, $\mu_{\mathrm{N}}$, was set to be close to $\mu_{\left[\mathrm{N}_{2}\right]}$ (i.e., the energy of $\mathrm{N}$ in a $\mathrm{N}_{2}$ molecule) to simulate the $\mathrm{N}$-rich growth condition in our work. Compared with the $\mathrm{N}$ atoms, the group-III metal atoms are the next-nearest (NN) atoms to the $\mathrm{Mg}$ substitutes. Since the number of $\mathrm{N}$ atoms can be regarded as constant, only the effect of the number change of $\mathrm{NN} G \mathrm{Ga}$ atoms on the $E^{\mathrm{f}}$ of $\mathrm{Mg}$ acceptors is considered, while the effect of the NN Al-atom number can be treated as the opposite. Fig. 2(a) and (b) show the $E^{\mathrm{f}}$ of $\mathrm{Mg}$ substitutes for $\mathrm{Ga}\left(\mathrm{Mg}_{\mathrm{Ga}}\right)$ and $\mathrm{Al}\left(\mathrm{Mg}_{\mathrm{Al}}\right)$ acceptors in the $\mathrm{Al}_{0.4} \mathrm{Ga}_{0.6} \mathrm{~N}$ bulk and at the $\mathrm{Al}_{0.8} \mathrm{Ga}_{0.2} \mathrm{~N}$ growth surface under $\mathrm{N}$-rich conditions as a function of the NN Ga-atom number. It can be seen that the $E^{\mathrm{f}}$ values of $\mathrm{Mg}_{\mathrm{Al}}$ and $\mathrm{Mg}_{\mathrm{Ga}}$ in the surface


Fig. 2 Formation energies of $\mathrm{Mg}_{\mathrm{Al}}$ and $\mathrm{Mg}_{\mathrm{Ga}}$ in the neutral charge state as a function of the $\mathrm{NN} \mathrm{Ga}$ atom numbers on the growth surface (a) and in the bulk (b) under $\mathrm{N}$-rich conditions. The supercell geometry of the $\mathrm{Al}_{0.4} \mathrm{Ga}_{0.6} \mathrm{~N}$ disordered alloy (c) and $\mathrm{Al}_{0.8} \mathrm{Ga}_{0.2} \mathrm{~N}$ disordered alloy (d) with one $\mathrm{Mg}$ substitution atom, surrounded by 3 next-nearest Ga atoms (as examples), which are labeled by black circles. 
are negative, suggesting favorable $\mathrm{Mg}$ substitution in this case. The $E^{\mathrm{f}}$ of $\mathrm{Mg}_{\mathrm{Al}}$ is lower than that of $\mathrm{Mg}_{\mathrm{Ga}}$ and increases as the NN Ga-atom number increases. In contrast to the surface case, $E^{\mathrm{f}}$ in the bulk is positive, which limits the $\mathrm{Mg}$ incorporation, as shown in Fig. 2(b). The $E^{\mathrm{f}}$ of $\mathrm{Mg}_{\mathrm{Al}}$ is larger than that of $\mathrm{Mg}_{\mathrm{Ga}}$ in the bulk of the $\mathrm{Al}_{0.4} \mathrm{Ga}_{0.6} \mathrm{~N}$ alloy, suggesting that the $\mathrm{Mg}$ atoms prefer to replace $\mathrm{Ga}$ sites rather than $\mathrm{Al}$ sites. It was also found that the $E^{\mathrm{f}}$ values of $\mathrm{Mg}_{\mathrm{Ga}}$ and $\mathrm{Mg}_{\mathrm{Al}}$ decrease monotonically with increasing $\mathrm{NN} \mathrm{Ga-atom} \mathrm{number} \mathrm{(i.e.} \mathrm{the} \mathrm{Ga} \mathrm{composition).}$ These characteristics can be mainly ascribed to the larger cohesive energy of the $\mathrm{Al}-\mathrm{N}$ bond $(2.88 \mathrm{eV})$ as compared to that of the $\mathrm{Ga}-\mathrm{N}$ bond $(2.24 \mathrm{eV}) .{ }^{25}$ Besides, it has been proposed that the $E^{\mathrm{f}}$ of a p-type dopant with a larger atomic size than the host atom increases with the increase of compressive stress. ${ }^{26}$ Therefore, the enhanced compressive strain, leading from $\mathrm{Mg}_{\mathrm{Al}}$ due to the smaller covalent radius of $\mathrm{Al}(1.18 \AA)$ than $\mathrm{Ga}(1.26 \AA)$ and the larger covalent radius of $\mathrm{Mg}(1.30 \AA)$, may also contribute to the larger $E^{\mathrm{f}}$ of $\mathrm{Mg}_{\mathrm{Al}}$. According to the above calculation results, the introduction of a pulsed $\mathrm{Ga}$ or $\mathrm{Al}$ source is expected to have a significant effect on the $\mathrm{Mg}$ incorporation at the $\delta$-doping plane. Therefore, the samples grown with the PTFG and PTAF were prepared together with the control sample for comparison.

Table 1 summarizes the Hall measurement results of the three p-type AlGaN samples determined at room temperature (RT). It can be found that the sample grown with the PTGF shows superior electrical properties over the control sample and the sample grown with the PTAF. A p-type conductivity with a high hole concentration $(p)$ of $8.30 \times 10^{18} \mathrm{~cm}^{-3}$, a low resistivity of $0.51 \Omega \mathrm{cm}$, and a hole mobility of $1.47 \mathrm{~cm}^{2} \mathrm{~V}^{-1} \mathrm{~s}^{-1}$ was achieved in the sample grown with the PTGF. The hole concentration was significantly increased, contributing most to the enhanced electrical conductivity with a resistivity comparable to those of the state-of-the-art p-GaN. ${ }^{27,28}$ Besides, the hole mobility of the PTGF sample is also slightly higher than that of the control sample, despite the higher hole concentration. This is likely due to the reduced dislocation scattering. The HRXRD rocking-curve width for the (0002) peak, which mainly reflects the screw-component dislocation density, decreases from 429 to 261 arcsec by the introduction of the PTGF. Compared with the control sample, the sample grown with the PTAF, however, demonstrated a remarkably lower hole concentration of $4.60 \times 10^{17} \mathrm{~cm}^{-3}$ and a consequently high resistivity of $5.38 \Omega \mathrm{cm}$. These results indicate that the pulsed precursors at the $\delta$-doping plane have an important effect on the hole concentration.

SIMS measurements were then performed to investigate the $\mathrm{Mg}$ incorporation in the different p-AlGaN samples. Fig. 3 depicts the SIMS depth profiles of $\mathrm{Mg}, \mathrm{Al}$ and $\mathrm{Ga}$ along the

Table 1 Results of Hall-effect measurements at RT

\begin{tabular}{llll}
\hline Sample & $\begin{array}{l}\text { Hole concentration } \\
\left(\mathrm{cm}^{-3}\right)\end{array}$ & $\begin{array}{l}\text { Mobility } \\
\left(\mathrm{cm}^{2} \mathrm{~V}^{-1} \mathrm{~s}^{-1}\right)\end{array}$ & $\begin{array}{l}\text { Resistivity } \\
(\Omega \mathrm{cm})\end{array}$ \\
\hline Control & $4.97 \times 10^{18}$ & 1.35 & 0.93 \\
W/PTGF & $8.30 \times 10^{18}$ & 1.47 & 0.51 \\
W/PTAF & $4.61 \times 10^{17}$ & 2.52 & 5.38
\end{tabular}
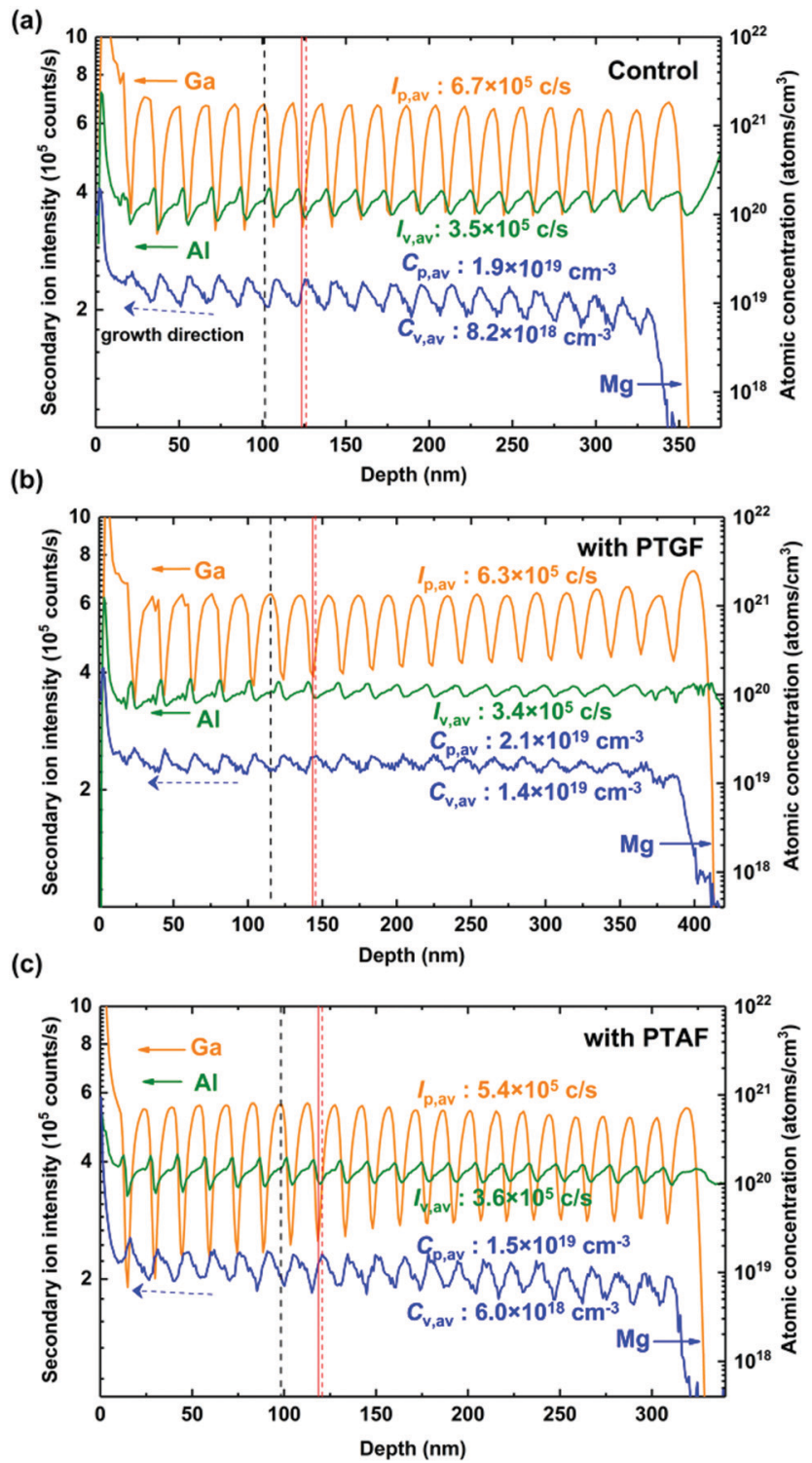

Fig. 3 SIMS depth profiles of Al, Ga, and Mg obtained from the (a) control, (b) PTGF, and (c) PTAF samples.

growth direction. Clear periodic oscillations were observed in the profile of the $\mathrm{Mg}$ atomic concentration $\left(C_{\mathrm{Mg}}\right)$ in the three samples, which are consistent with the feature of $\delta$-doping. The depth profiles of the $\mathrm{Al}$ and $\mathrm{Ga}$ intensity also show oscillations with the same period as $C_{\mathrm{Mg}}$, which are ascribed to the preferential dissociation of $\mathrm{Ga}-\mathrm{N}$ bonds during the purge step and the substitution of $\mathrm{Mg}$ at $\mathrm{Al}$ and $\mathrm{Ga}$ sites. As a result of the dissociation, the $\mathrm{Ga}$ intensity decreases to the valley point of the oscillation profile, which can be recognized as the positions of the $\delta$-doping plane (e.g., the vertical red line in Fig. 3a). During the $\delta$-doping process, the $\mathrm{Mg}$ atoms not only occupy group III vacancies generated by In and Ga desorption during the purge step, but also tend to replace $\mathrm{Al}$ atoms due to the lower $E^{\mathrm{f}}$ of $\mathrm{Mg}_{\mathrm{Al}}$ on the $\mathrm{Al}_{x} \mathrm{Ga}_{1-x} \mathrm{~N}$ surface. Consequently, it is shown that the peak positions of the $C_{\mathrm{Mg}}$ profile (the vertical red dashed line) correspond to the valley positions of the 
Al-intensity profile, which slightly deviated from the $\delta$-doping plane backward. The backward diffusion of the $\delta$-doped $\mathrm{Mg}$ atoms is accompanied by $\mathrm{Mg}$ substitution on $\mathrm{Ga}$ and $\mathrm{Al}$ sites, leading $C_{\mathrm{Mg}}$ to reach a valley point between two $\delta$-doping planes. In this process, the attenuation of $C_{\mathrm{Mg}}$ mainly depends on the substitution of $\mathrm{Ga}$ by $\mathrm{Mg}$. This is because the $E^{\mathrm{f}}$ of $\mathrm{Mg}_{\mathrm{Ga}}$ is lower than that of $\mathrm{Mg}_{\mathrm{Al}}$ in the bulk case, i.e. substituting $\mathrm{Mg}$ for $\mathrm{Ga}$ is more energetically favorable. Therefore, the valley point of the $C_{\mathrm{Mg}}$ profile corresponds to the peak position of the Ga-intensity profile, indicated as the vertical black dashed line shown in Fig. 3a.

Moreover, it is notable that the depth profile of $C_{\mathrm{Mg}}$ shows an upward slope along the growth direction of the control sample. According to the results shown in Fig. 2, when the growing surface is covered by newly added layers during the epitaxy, the inner $\mathrm{Mg}$ atoms tend to segregate to the new surface to regain equilibrium because of the lower $E_{\mathrm{f}}$ of $\mathrm{Mg}$ substitutes on the surface, while a part of $\mathrm{Mg}$ substitutes remain in the layer that has been converted into the bulk. Consequently, $C_{\mathrm{Mg}}$ increases gradually along the growth direction. This is the case for the forward diffusion and incorporation of $\delta$-doped $\mathrm{Mg}$ atoms, which is dominated by the competition between the substitution of $\mathrm{Al}$ by $\mathrm{Mg}$ on the growing surface and the substitution of $\mathrm{Ga}$ by $\mathrm{Mg}$ in the bulk.

For the PTGF sample, the entire incorporation of $\mathrm{Mg}$ is significantly enhanced, as illustrated in Fig. 3b. The average $C_{\mathrm{Mg}}$, calculated by integrating the depth profile of $C_{\mathrm{Mg}}$ over the p-AlGaN layer and dividing the integral by the layer thicknesses, is $1.6 \times 10^{19} \mathrm{~cm}^{-3}$, about 1.4 times higher than that of the control sample and indicating a very high doping efficiency $\eta_{\mathrm{p}}$ $\left(=p / C_{\mathrm{Mg}}\right)$ of $51.9 \%$. It was found that the average peak and valley values of the $C_{\mathrm{Mg}}$ profile $\left(C_{\mathrm{p} \text {,av }}\right.$ and $\left.C_{\mathrm{v}, \text { av }}\right)$ are $2.1 \times$ $10^{19}$ and $1.4 \times 10^{19}$, respectively; both are higher than the corresponding values of the control sample. Meanwhile, the Al and Ga profiles show a decrease in the average valley and peak intensities $\left(I_{\mathrm{v}, \mathrm{av}}\right.$ and $\left.I_{\mathrm{p}, \mathrm{av}}\right)$, respectively, as compared to the control sample. This means that the $\mathrm{Mg}$ substitution for $\mathrm{Al}$ is enhanced by introducing the PTGF at the $\delta$-doping planes and the substitution for $\mathrm{Ga}$ is also increased in the layer between the $\delta$-doping planes.

It can be reasoned that the supplement of Ga atoms by the use of the PTGF prevents the $\delta$-doped $\mathrm{Mg}$ from diffusing into the interface of the next growth layer due to the much higher $E^{\mathrm{f}}$ of $\mathrm{Mg}_{\mathrm{Ga}}$ compared to that of $\mathrm{Mg}_{\mathrm{Al}}$ on the surface of the grown layer. The $\mathrm{Mg}$ incorporation on the underlayer surface is therefore enhanced, leading to a slightly higher value of $C_{\mathrm{p} \text {,av }}$. On the other hand, the PTGF also makes a small amount of Ga incorporate into the next growth layer, which is evidenced by the enhanced $\mathrm{Ga}$ intensities at the valley points and the increased growth rate with respect to the control sample. The increase of $\mathrm{Ga}$ incorporation reduces the $E^{\mathrm{f}}$ of $\mathrm{Mg}_{\mathrm{Ga}}$ and $\mathrm{Mg}_{\mathrm{Al}}$ in the bulk, especially the $E^{\mathrm{f}}$ of $\mathrm{Mg}_{\mathrm{Ga}}$, and thus increases the $\mathrm{Mg}$ incorporation. As a result, the value of $C_{\mathrm{v}, \mathrm{av}}$ is significantly increased and the $I_{\mathrm{p} \text {,av }}$ of the Ga intensity profile is correspondingly decreased. Such an Mg-incorporation enhancement in the bulk also suppresses the $\mathrm{Mg}$ segregation to the growing surface, leading to a $C_{\mathrm{Mg}}$ profile without inclination as shown in Fig. $3 \mathrm{~b}$.

On the contrary, as shown in Fig. 3c, the $\mathrm{Mg}$ incorporation is inhibited markedly by introducing the PTAF. Compared with the control sample, the $I_{\mathrm{v} \text {,av }}$ value of the Al profile increases, while the $I_{\mathrm{p} \text {,av }}$ value of the Ga profile decreases for the PTAF sample. The decrease of $I_{\mathrm{p} \text {,av }}$ should be due to the lowered $\mathrm{Ga}$ incorporation by introducing the PTAF, rather than $\mathrm{Mg}$ substituting $\mathrm{Ga}$ in the layer, because the corresponding $C_{\mathrm{v} \text {,av }}$ of the $\mathrm{Mg}$ profile is also decreased. It has been reported that the incorporation of $\mathrm{Ga}$ is generally blocked by the $\mathrm{Al}$ incorporation during the growth of AlGaN, because competitive adsorption of $\mathrm{Al}$ atoms hinders the incorporation of $\mathrm{Ga}$ atoms. ${ }^{29}$ The above argument is also supported by the observed reduction in the growth rate (i.e., thickness) of the PTAF sample (Fig. 3c).

According to the change of $C_{\mathrm{Mg}}$ and the corresponding changes of the $\mathrm{Al}$ and $\mathrm{Ga}$ intensity, the mechanism for the reduced $\mathrm{Mg}$ incorporation in this case can be interpreted in terms of the diversion effect of the PTAF on the $\delta$-doped $\mathrm{Mg}$ and its blocking effect on the $\mathrm{Mg}_{\mathrm{Ga}}$ formation. Under the extremely Al-rich condition with the use of the PTAF, the $\delta$-doped $\mathrm{Mg}$ diffused to the surface of the grown layer is diverted to the surface of the growing layer, resulting in a reduction of $C_{\mathrm{p} \text {,av }}$ at the $\delta$-doping planes. Although the growing surface shares the doped $\mathrm{Mg}$ due to the lower $E^{\mathrm{f}}$ of $\mathrm{Mg}_{\mathrm{Al}}$, in the bulk layer transformed from the surface, the $\mathrm{Mg}$ incorporation decreases significantly due to the higher $E^{\mathrm{f}}$ of $\mathrm{Mg}_{\mathrm{Ga}}$ and $\mathrm{Mg}_{\mathrm{Al}}$ caused by the increase of $\mathrm{Al}$ incorporation. The incorporation of $\mathrm{Mg}$ is thus reduced overall. In addition, the segregation of $\mathrm{Mg}$ was also improved due to the diversion effect of the PTAF at the $\delta$-doping planes. However, as the $\mathrm{Mg}$ incorporation in the bulk layer has not been enhanced, the effect of segregation inhibition is not as significant as that of the PTGF.

Besides the $\mathrm{Mg}$ incorporation, the activation energy $\left(E_{\mathrm{A}}\right)$ of the $\mathrm{Mg}$ acceptor is also a key factor in enhancing the doping efficiency. Temperature-dependent Hall-effect measurements were therefore performed on the PTGF and control samples from $300 \mathrm{~K}$ to $560 \mathrm{~K}$ in $15 \mathrm{~K}$ increments. Fig. 4 presents the Arrhenius plots of hole concentration versus reciprocal temperature for the two samples. The value of $E_{\mathrm{A}}$, the acceptor concentration $\left(N_{\mathrm{A}}\right)$, and the compensating donor concentration $\left(N_{\mathrm{D}}\right)$ can be extracted by fitting the data points in the Arrhenius plot to the formula

$$
\frac{p\left(p+N_{\mathrm{D}}\right)}{N_{\mathrm{A}}-N_{\mathrm{D}}-p}=\frac{N_{\mathrm{V}}}{g} \exp \left(-\frac{E_{\mathrm{A}}}{k T}\right)
$$

where $p$ is the total hole concentration, $k$ the Boltzmann constant, $T$ the temperature, $g$ the acceptor degeneracy, which is assumed to be equal to 4 , and $N_{\mathrm{V}}$ the effective valence band state density of $N_{\mathrm{V}}=2\left(2 \pi m_{\mathrm{h}}{ }^{*} k T\right)^{3 / 2} / h^{3}$. ${ }^{30}$ The results show that $E_{\mathrm{A}}$ decreases from $39 \pm 3 \mathrm{meV}$ to $26 \pm 3 \mathrm{meV}$ by introducing the PTGF. Although a reduction in the band gap by the enhanced Ga incorporation may contribute to this decrease, its impact should be small. According to the approximate dependence of the $\mathrm{Mg}$ activation energy on the $\mathrm{Al}$ composition of AlGaN, ${ }^{9}$ $E_{\mathrm{A}}$ decreases by $\sim 1 \%$ when the $\mathrm{Al}$ composition decreases from 


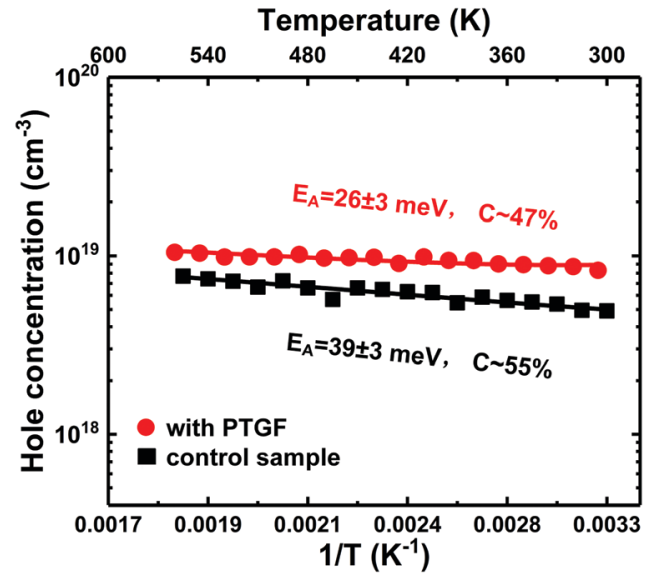

Fig. 4 Temperature-dependent hole concentration for the PTGF and control samples. The fitting curves are shown as solid lines.

$42.4 \%$ to $41.7 \%$ (as will be shown below), much smaller than the $33 \%$ reduction of $E_{\mathrm{A}}$ in this case. Moreover, by using $N_{\mathrm{A}}$ determined from the SIMS data, the compensation ratio $C$ $\left(N_{\mathrm{D}} / N_{\mathrm{A}}\right)$ for the PTGF sample was obtained as $\sim 47 \%$, lower than the $\sim 55 \%$ for the control sample, which suggests a reduction of the self-compensation effect.

The very low values of $E_{\mathrm{A}}$ could be due to the strong band modulation (i.e., composition modulation) effect resulting from the delta-doping process, as shown in Fig. 3. It was found from the SIMS data that the $\mathrm{Al}$ compositions around the $\delta$-doping plane are higher, leading to a self-formed periodic structure with a quantum well (QW) and a graded barrier (GB). In order to clarify the effect of band modulation on the lowered $E_{\mathrm{A}}$ by introducing the PTGF, we simulated the valence band profiles along the growth direction for the control and PTGF samples by self-consistently solving the 1D coupled Schrödinger-Poisson equations with the TCAD tool Silvaco Atlas. In the simulations, the depth profiles of $\mathrm{Al}$ and $\mathrm{Ga}$ compositions were deduced from the SIMS results and verified by fitting the XRD $2 \theta-\omega$ scan curves of the (0002) reflection. The thicknesses of the QW and GB regions were estimated to be $\sim 12.5$ and $5.7 \mathrm{~nm}$, and 13.9 and $6.8 \mathrm{~nm}$ for the control and PTGF samples, respectively, while the Al compositions of the QW and GB peak were $\sim 42.4 \%$ and $81 \%$, and $41.7 \%$ and $80.8 \%$, respectively. The increased thicknesses of the QW and GB regions of the $\mathrm{p}$-AlGaN layer grown with the PTGF are ascribed to the increased incorporation of Ga atoms. Also, the enhanced incorporation contributes to the lower minimum of the Al composition (Fig. 5).

The resulting valence band diagrams are shown in Fig. 6 . As seen in Fig. 6a and b, the valence band bends upward at the QW/GB interface (dashed ring) due to the strong internal polarization fields in the III-nitrides. ${ }^{31}$ Consequently, the holes in the two p-AlGaN samples are contributed by the following parts: (1) the thermally ionized holes arising from the QW regions where the $\mathrm{Mg}$ acceptor level $\left(E_{\mathrm{Mg}}\right)$ is above the Fermi level (almost no holes from the acceptors in the GB regions due to the high ionization energy); (2) the holes released from the

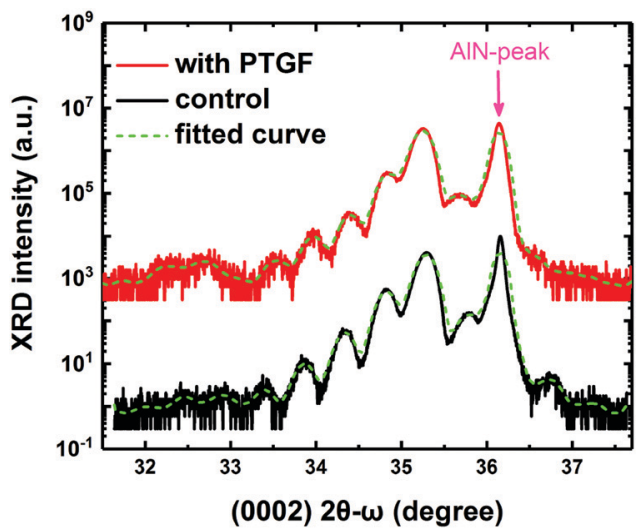

Fig. 5 HRXRD (0002)-plane $2 \theta-\omega$ scan curves for the PTGF sample and control sample.

$\mathrm{Mg}$ acceptors in the QW and GB regions where $E_{\mathrm{Mg}}$ is below the Fermi level; and (3) the holes accumulated at the QW/GB interfaces where the valence band bends and exceeds the Fermi level, forming a two-dimensional hole gas (2DHG). The quantity of holes from parts (1) and (2) should be increased in the case of doping with the PTGF, because of the significantly increased $C_{\mathrm{Mg}}$, which is mainly contributed by the increment of $\mathrm{Mg}_{\mathrm{Ga}}$ acceptors with lower $E^{\mathrm{f}}$ in part (1) and by the increment of $\mathrm{Mg}_{\mathrm{Al}}$ acceptors in part (2). In part (3), more $2 \mathrm{DHG}$ is expected to accumulate at the heterointerface of the $\delta$-doping layer grown with the PTGF, due to the extended interfacial band bending area as shown in Fig. 6c. Also shown in Fig. $6 b$ and $c$ is the valence band of the control sample, in which the Al compositions of the peak and valley remain unchanged, while the thicknesses of the QW and GB become the same as those of the PTGF sample. It can be seen that the band bending of the PTGF sample is still slightly deeper at the QW/GB interfaces, indicating that the band bending mainly results from the polarization field introduced by the change of the Al composition, rather than the thickness change of the QW and GB.

Besides, it should be noted that the $E_{\mathrm{A}}$ of the PTGF sample may also be lowered by the enhanced Coulomb interaction between the ionized acceptors and the increased screening of the Coulomb potential by the free carriers. ${ }^{32,33}$ The enlarged band bending combined with the increased acceptor concentration led to a much lower equivalent $E_{\mathrm{A}}$ value in the PTGF sample.

The optical transmission spectra of the three samples were also recorded under the normal-incidence condition. As shown in Fig. 7a, the transmittance $(T)$ of the transmission peaks is larger than $80 \%$ at wavelengths above the absorption edge of the three thermally-annealed p-AlGaN samples, even without considering the reflection loss. Such high transmittance is beneficial for DUV optoelectronic applications. Assuming the absorption coefficient $\alpha \sim \ln (1 / T)$ corresponding to the direct energy band gap $\left(E_{\mathrm{g}}\right)$ of AlGaN, the equivalent $E_{\mathrm{g}}$ of our p-AlGaN layers with QW and QB structure can be estimated by extrapolating the linear portion of the $(\alpha h \nu)^{2}$ versus photon energy $(h \nu)$ plot. The obtained values of $E_{\mathrm{g}}$ are $4.43,4.47$, and $4.39 \mathrm{eV}$ for the 
(a)

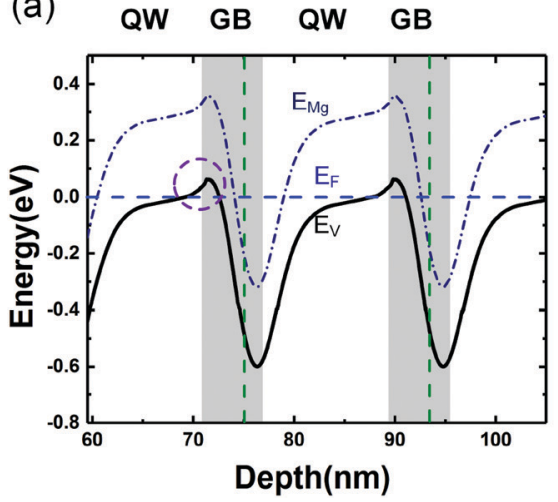

(b)

QW GB QW GB

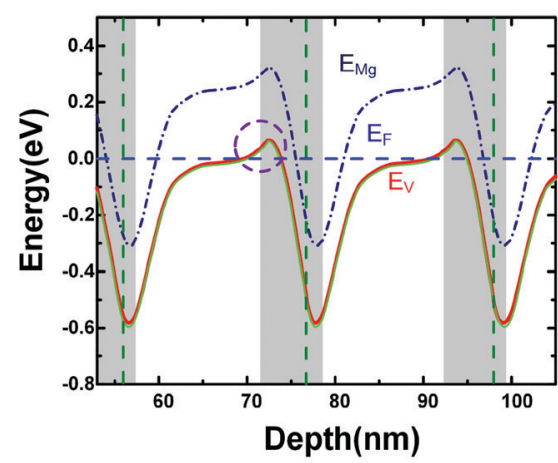

(c)



Fig. 6 The simulated valence band diagrams of the control (a) and the PTGF (b) samples. The vertical green dashed lines indicate the positions of $\delta$-doping planes. A detailed diagram for comparing the valence band profiles around the QW/GB interface is shown in (c). The green lines in (b) and (c) are the valence band of the control sample with the thicknesses of the QW and GB consistent with those of the PTGF sample.
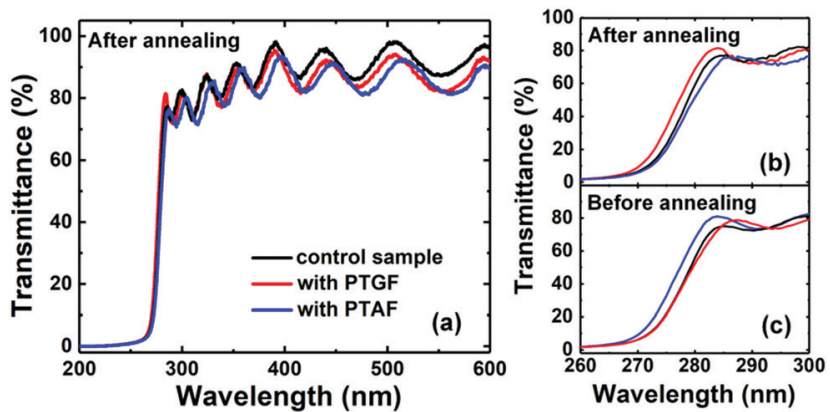

Fig. 7 (a) Spectral transmittances for the control, PTGF, and PTAF samples after the thermal annealing. Transmittance spectra around the absorption edge for the three samples (b) after and (c) before the thermal annealing.

control, PTGF, and PTAF samples, respectively. These values are contrary to those expected from the $\mathrm{Al}$ compositions of the three samples, in which the PTAF sample has the largest Al composition of $43.6 \%$, while the PTGF sample has the smallest one of $41.7 \%$. We ascribed this result to the Burstein-Moss (band filling) effect due to the high hole concentrations in the control and PTGF samples. It was shown that before the thermal activation, the $E_{\mathrm{g}}$ values are $4.39,4.38$, and $4.48 \mathrm{eV}$ for the control, PTGF, and PTAF samples (Fig. 7c), respectively, indicating that the blue shift of the thermally-activated control and PTGF samples stems from the band filling effect induced by the degenerate p-type doping.

Since a pn junction is the basic building block of most diodes and transistors, the formation of a high-quality Al-rich AlGaN pn junction is crucial to verify the applicability of our p-type AlGaN layer grown with the PTGF. Therefore, Al-rich AlGaN pn junction diodes were fabricated using the $\mathrm{Mg}-\delta$ doped $\mathrm{Al}_{x} \mathrm{Ga}_{1-x} \mathrm{~N}(x \sim 0.42)$ grown with the PTGF. The epitaxial structure and fabrication process were as described in the Experimental section. Fig. 8 gives the typical current-voltage $(I-V)$ characteristics of the fabricated pn junction diodes. As shown in Fig. 8a, the pn junction with a $100 \mu \mathrm{m}$ diameter active area exhibited a low leakage current of less than $5 \times 10^{-12}$ A when the reverse biases were below $10 \mathrm{~V}$. The low reverse leakage current implies high-quality junction interfaces and relatively low screw-component dislocation density (the primary leakage path in III-nitride semiconductors) in the AlGaN pn diodes. A screw dislocation density of $\sim 1.67 \times$ $10^{8} \mathrm{~cm}^{-2}$ was estimated from the XRD rocking curve linewidth for the symmetric AlGaN (0002) diffraction peak. Under the
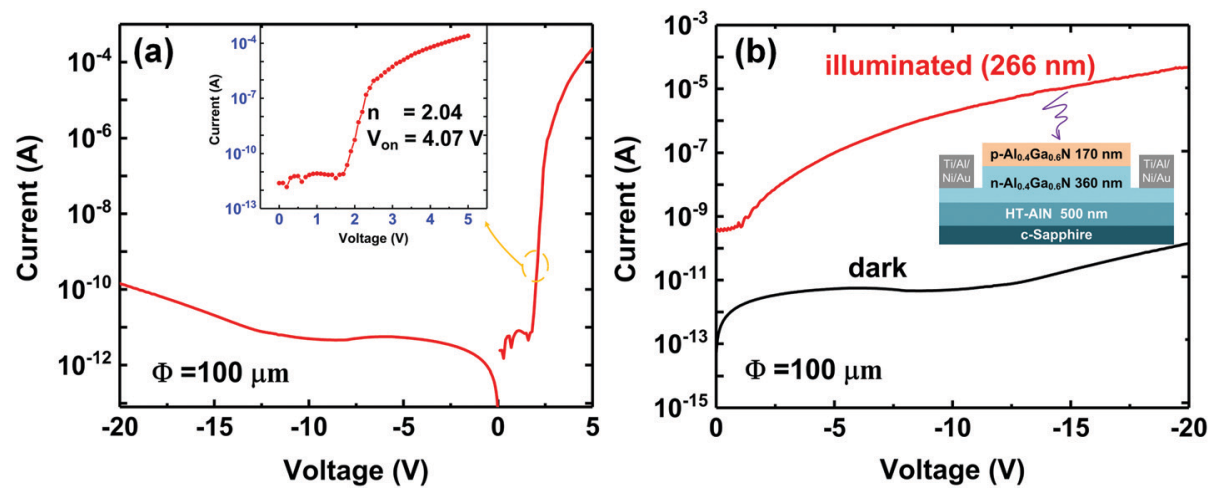

Fig. 8 (a) Dark $I-V$ curve of the full Al-rich AlGaN p-n junction diode with the $p$-type layer grown with the PTGF. (b) Reverse $I-V$ curves of the diode with a mesa diameter of $100 \mu \mathrm{m}$ under the dark and UV illumination conditions. The insets show the forward $I-V$ curve and the schematic structure of the pn junction. 
forward bias condition, the current is $\sim 0.1 \mathrm{~mA}$ at $5 \mathrm{~V}$ bias with a turn on voltage of $\sim 4.07 \mathrm{~V}$, as expected from the band gap of $\mathrm{Al}_{x} \mathrm{Ga}_{1-x} \mathrm{~N}(x \sim 0.42)$. An ideality factor $(n)$ of 2.04 was obtained by fitting the forward $I-V$ curve in the intermediate bias region with the diode equation

$$
I=I_{0}[\exp (q V / n k T)-1]
$$

where $I_{0}$ is the reverse saturation current, and $q$ is the charge of the electron. The value of $n$ close to 2 suggests that the forward current is dominated by the generation-recombination process. These results thus demonstrate the formation of a high quality full Al-rich AlGaN pn junction.

Moreover, the photoresponse of the AlGaN diodes was also investigated. A DUV light emitting diode with a center wavelength of $266 \mathrm{~nm}$ was employed as a light source. The incident optical power density was $220 \mu \mathrm{W} \mathrm{cm} \mathrm{cm}^{-2}$, as determined by a Newport power meter. Under front UV illumination, the strong photoresponse again verifies that the fabricated AlGaN diodes are pn junctions rather than the Schottky barrier diode formed by the $\mathrm{Ni} / \mathrm{Au}$ electrode on $\mathrm{p}$-AlGaN, since the photocurrent generated from the depletion region of a zero-biased opaque Schottky contact should be much smaller than that generated from the space-charge region of a pn junction, as in this case (Fig. 8b). The significant photocurrent also indicates the feasibility of the p-AlGaN grown with the PTGF in AlGaN based DUV optoelectronics.

In summary, high doping efficiency has been achieved in $\mathrm{Mg}$ - $\delta$-doped Al-rich AlGaN. It was shown by DFT calculations that the $\mathrm{Mg}$ substitution is more energetically favorable on the surface of AlGaN, especially for the substitution on the Al site, whereas in the bulk layer the doped $\mathrm{Mg}$ prefers to substitute the Ga site. Accordingly, a pulsed TMGa flow was introduced to modify the Mg-doping planes, in which the $\delta$-doping plane can be classified into upper and lower surfaces. In the process of growth, the lower surface is modified by the purge step to increase the Al composition, while the upper surface is modified by the PTGF to impede the upward diffusion of the $\mathrm{Mg}$ dopant. Meanwhile, as the epitaxial growth continues, the upper surface is transformed into the bulk of the underlayer with a slightly higher Ga composition by the PTGF, which leads to enhanced $\mathrm{Mg}$ incorporation in the bulk. A high hole concentration of $8.3 \times 10^{18} \mathrm{~cm}^{-3}$ was therefore obtained in $\mathrm{Al}_{x} \mathrm{Ga}_{1-x} \mathrm{~N}$ $(x \sim 0.42)$ with an average $\mathrm{Mg}$ doping concentration of $1.6 \times$ $10^{19}$, indicating a doping efficiency of up to $51.9 \%$. The resistivity is as low as $0.51 \Omega \mathrm{cm}$, comparable to those of the-state-of-art p-GaN. Also, the prepared p-type layer is highly transparent at wavelengths above the absorption edge. The SIMS results show that the $\mathrm{Mg}$ incorporation using the proposed method was enhanced by 1.4 times, which can be ascribed to the enhanced $\mathrm{Mg}$ substitution for $\mathrm{Al}$ by introducing the PTGF at the $\delta$-doping planes and the increased substitution for $\mathrm{Ga}$ in the bulk layer. Temperature-dependent Hall measurements indicate a very small $E_{\mathrm{A}}$ of $\sim 26 \mathrm{meV}$, much lower than the $39 \mathrm{meV}$ of the control sample. The decrease in $E_{\mathrm{A}}$ is attributed to the enlarged valence-band bending by the PTGF. Moreover, Al-rich AlGaN pn junctions were fabricated using the p-AlGaN layer grown with the modified doping planes.
The resulting pn junctions demonstrate excellent characteristics in terms of leakage current, turn-on voltage, and ideality factor, which proves the applicability of the doping-plane modification concept.

\section{Conflicts of interest}

There are no conflicts to declare.

\section{Acknowledgements}

This work was supported by the National Key R\&D Program of China (Grant No. 2016YFB0400901), the State Key Program of National Natural Science Foundation of China (Grant No. 61634002), and Key Realm R\&D Program of GuangDong Province (Grant No. 2019B010132004).

\section{References}

1 M. L. Nakarmi, N. Nepal, J. Y. Lin and H. X. Jiang, Appl. Phys. Lett., 2009, 94, 091903.

2 K. B. Nam, J. Li, M. L. Nakarmi, J. Y. Lin and H. X. Jiang, Appl. Phys. Lett., 2002, 81, 1038-1040.

3 J. P. Zhang, X. Hu, Y. Bilenko, J. Deng, A. Lunev, M. S. Shur, R. Gaska, M. Shatalov, J. W. Yang and M. A. Khan, Appl. Phys. Lett., 2004, 85, 5532-5534.

4 Z. G. Shao, D. J. Chen, H. Lu, R. Zhang, D. P. Cao, W. J. Luo, Y. D. Zheng, L. Li and Z. H. Li, IEEE Electron Device Lett., 2014, 35, 372-374.

5 T. Kinoshita, T. Obata, H. Yanagi and S.-I. Inoue, Appl. Phys. Lett., 2013, 102, 012105.

6 Z. Jun, T. Wu, W. Feng, Y. Weiyi, X. Hui, D. Jiangnan, F. Yanyan, W. Zhihao and C. Changqing, IEEE Photonics J., 2013, 5, 1600310.

7 J. Simon, V. Protasenko, C. Lian, H. Xing and D. Jena, Science, 2010, 327, 60-64.

8 T. M. Al tahtamouni, J. Y. Lin and H. X. Jiang, AIP Adv., 2014, 4, 047122.

9 Y. H. Liang and E. Towe, Appl. Phys. Rev., 2018, 5, 011107.

10 S. R. Jeon, Z. Ren, G. Cui, J. Su, M. Gherasimova, J. Han, H. K. Cho and L. Zhou, Appl. Phys. Lett., 2005, 86, 082107.

11 W. Luo, B. Liu, Z. Li, L. Li, Q. Yang, L. Pan, C. Li, D. Zhang, X. Dong, D. Peng, F. Yang and R. Zhang, Appl. Phys. Lett., 2018, 113, 072107.

12 M. Martens, C. Kuhn, E. Ziffer, T. Simoneit, V. Kueller, A. Knauer, J. Rass, T. Wernicke, S. Einfeldt, M. Weyers and M. Kneissl, Appl. Phys. Lett., 2016, 108, 151108.

13 C. Bayram, J. L. Pau, R. McClintock and M. Razeghi, J. Appl. Phys., 2008, 104, 083512.

14 J. Li, W. Yang, S. Li, H. Chen, D. Liu and J. Kang, Appl. Phys. Lett., 2009, 95, 151113.

15 J. Tersoff, Phys. Rev. Lett., 1995, 74, 5080-5083.

16 G. Kresse and J. Furthmuller, Phys. Rev. B: Condens. Matter Mater. Phys., 1996, 54, 11169-11186.

17 G. Kresse and J. Furthmüller, Comput. Mater. Sci., 1996, 6, 15-50. 
18 P. E. Blöchl, Phys. Rev. B: Condens. Matter Mater. Phys., 1994, 50, 17953-17979.

19 J. P. Perdew, K. Burke and M. Ernzerhof, Phys. Rev. Lett., 1996, 77, 3865-3868.

20 H. J. Monkhorst and J. D. Pack, Phys. Rev. B: Solid State, 1976, 13, 5188-5192.

21 C. G. Van de Walle and J. Neugebauer, J. Appl. Phys., 2004, 95, 3851-3879.

22 A. Zoroddu, F. Bernardini, P. Ruggerone and V. Fiorentini, Phys. Rev. B: Condens. Matter Mater. Phys., 2001, 64, 045208.

23 B. Lange, C. Freysoldt and J. Neugebauer, Phys. Rev. B: Condens. Matter Mater. Phys., 2010, 81, 2583-2587.

24 Y. Chen, H. Wu, E. Han, G. Yue, Z. Chen, Z. Wu, G. Wang and H. Jiang, Appl. Phys. Lett., 2015, 106, 162102.

25 W. J. O.-T., J. Mol. Struct., 1981, 71, 355.

26 J. Zhu, F. Liu, G. B. Stringfellow and S. H. Wei, Phys. Rev. Lett., 2010, 105, 195503.
27 E. C. H. Kyle, S. W. Kaun, E. C. Young and J. S. Speck, Appl. Phys. Lett., 2015, 106, 222103.

28 Y. Chen, H. Wu, G. Yue, Z. Chen, Z. Zheng, Z. Wu, G. Wang and H. Jiang, Appl. Phys. Express, 2013, 6, 041001.

29 D. G. Zhao, Z. S. Liu, J. J. Zhu, S. M. Zhang, D. S. Jiang, H. Yang, J. W. Liang, X. Y. Li and H. M. Gong, Appl. Surf. Sci., 2006, 253, 2452-2455.

30 T. Tanaka, A. Watanabe, H. Amano, Y. Kobayashi, I. Akasaki, S. Yamazaki and M. Koike, Appl. Phys. Lett., 1994, 65, 593-594.

31 T. Takeuchi, C. Wetzel, S. Yamaguchi, H. Sakai, H. Amano, I. Akasaki, Y. Kaneko, S. Nakagawa, Y. Yamaoka and N. Yamada, Appl. Phys. Lett., 1998, 73, 1691-1693.

32 Y. Arakawa, K. Ueno, A. Kobayashi, J. Ohta and H. Fujioka, APL Mater., 2016, 4, 086103.

33 B. Sarkar, S. Mita, P. Reddy, A. Klump, F. Kaess, J. Tweedie, I. Bryan, Z. Bryan, R. Kirste, E. Kohn, R. Collazo and Z. Sitar, Appl. Phys. Lett., 2017, 111, 032109. 